\title{
ON A RECENT THEOREM BY H. REITER
}

\section{NAMIOKA}

Let $G$ be a locally compact group with a fixed left invariant Haar measure $\mu$. Let us consider the following three statements concerning $G$. (All the linear spaces appearing below are real linear spaces.)

(M): There is a mean $m$ on $L_{\infty}(G)$ such that $m\left({ }_{x} f\right)=m(f)$ for each $f \in L_{\infty}(G)$ and each $x \in G$. Here a mean on $L_{\infty}(G)$ is a linear functional $m$ on $L_{\infty}(G)$ such that $m(g) \geqq 0$ whenever $g \geqq 0$ and $m(1)=1$, and, for a real valued function $f$ on $G,{ }_{x} f$ is a function on $G$ defined by ${ }_{x} f(y)$ $=f(x y)$.

$\left(\mathrm{P}_{1}\right)$ : Given a positive number $\epsilon$ and a compact subset $K$ of $G$, there is an element $s$ in $\Phi=\left\{f: f \in L_{1}(G), f \geqq 0\right.$ and $\left.\int f d \mu=1\right\}$ such that $\left\|{ }_{x} s-s\right\|_{1}<\epsilon$ for each $x \in K$.

$(\mathrm{J})$ : There is a mean $m$ on $L_{\infty}(G)$ such that $m(s * f)=m(f)$ for each $f \in L_{\infty}(G)$ and each $s \in \Phi$. Here "*" denotes the usual convolution with respect to $\mu$ (see, for instance, Hewitt-Ross [1]).

The property $(\mathrm{J})$ was introduced recently by Hulanicki in [2], where it is proved that a group $G$ satisfies $(\mathrm{J})$ if and only if it satisfies $\left(P_{1}\right)$. More recently Reiter proved that $(M)$ implies $\left(P_{1}\right)$ [3]. (The reverse implication $\left(\mathrm{P}_{1}\right) \Rightarrow(\mathrm{M})$ is simple and well known.) In this note we shall give a short proof of the implication $(M) \Rightarrow(J)$, thus giving another proof to Reiter's theorem.

TheOREM. If a locally compact group $G$ satisfies (M), then it satisfies $(\mathrm{J})$.

Proof. Let $m$ be a mean on $L_{\infty}(G)$ such that $m\left({ }_{x} f\right)=m(f)$ for each $f \in L_{\infty}(G)$ and each $x \in G$. Let $h$ be a fixed member of $L_{\infty}(G)$ such that $h \geqq 0$, and let $\phi$ be a linear functional on $L_{1}(G)$ defined by $\phi(g)=m(g * h)$ for $g \in L_{1}(G)$. Then, since $|\phi(g)|=|m(g * h)| \leqq\|g * h\|_{\infty}$ $\leqq\|g\|_{1} \cdot\|h\|_{\infty}, \phi$ is bounded, and $\phi\left({ }_{x} g\right)=\phi(g)$ for each $x \in G$ because of ${ }_{x} g * h={ }_{x}(g * h)$. Clearly $g \geqq 0$ implies $\phi(g) \geqq 0$. Therefore, by the uniqueness of Haar integral, there is a nonnegative number $k(h)$ such that, for each $g$ in $L_{1}(G)$,

$$
m(g * h)=k(h) \int g d \mu .
$$

Obviously $k(1)=1, k(\lambda h)=\lambda k(h)$ and $k\left(h+h^{\prime}\right)=k(h)+k\left(h^{\prime}\right)$ for $\lambda \geqq 0$ and nonnegative elements $h, h^{\prime}$ of $L_{\infty}(G)$. Hence $k$ can be extended to

Received by the editors February 28, 1966. 
be a mean (again denoted by $k$ ) on $L_{\infty}(G)$, and (1) is now valid for each $g \in L_{1}(G)$ and each $h \in L_{\infty}(G)$. Now take $s$ in $\Phi$ and $f$ in $L_{\infty}(G)$; then by (1) we have

$$
\begin{aligned}
k(f) & =k(f) \int s * s d \mu=m((s * s) * f)=m(s *(s * f)) \\
& =k(s * f) \int s d \mu=k(s * f) .
\end{aligned}
$$

Hence $k$ is a mean satisfying $(\mathrm{J})$.

\section{REFERENCES}

1. E. Hewitt and K. A. Ross, Abstract harmonic analysis, Vol. 1, Springer-Verlag, Berlin, 1963.

2. A. Hulanicki, Means and Folner condition on locally compact groups, Studia Math. (to appear).

3. H. Reiter, On some properties of locally compact groups, Indag. Math. 27 (1965), 697-701.

UNIVERSITY OF WASHINGTON 